\title{
Development of Absolute Magnetic Rotary Encoder with Eccentric Rotation
}

\author{
Kodai Yamamoto* Non-member, Kazuki Otomo* Non-member \\ Hideki Hashimoto* Senior Member
}

\begin{abstract}
To improve the resolution of a magnetic absolute encoder, we propose a new magnetic encoder with an eccentric structure. Since the proposed magnetic encoder consists of only one eccentrically rotating multi-pole magnet and four Hall elements, its structure is very simple and cost efficient. By calculating the absolute offset by combining the lookup table and least squares method, it is possible to calculate the absolute angle even when a multipolar magnet is used for resolution improvement. Furthermore, since the quadrature signal can be calculated, it is possible to configure the converter using the conventional method, and it is very versatile. Experimental results show that the proposed method can achieve both improvements of accuracy by increasing the number of pole pairs and calculation of absolute angle.
\end{abstract}

Keywords: magnetic encoder, absolute encoder

\section{Introduction}

High-precision motor control is an important technology in fields ranging from robotics and cars to automation devices. The precision of motor control depends on the angle and angular velocity obtained from an encoder, making reliable, high-precision encoders crucial for high-precision control. There is also increasing demand to make control systems smaller and smaller so that they can be included in any environment, which has in turn increased the demand for highprecision encoders that are also small.

Currently, the most widely used encoders are optical encoders $^{(1)-(3)}$. While optical encoders make it comparatively easy to achieve high angular resolution, they do not cope well with garbage, dust, and oil, making them unreliable and not very environment-tolerant. In addition, because optical encoders' slit widths are limited by physical constraints, the only way to increase angular resolution is to increase the size of the encoder. Obtaining its absolute angle requires a further increase in size.

Meanwhile, magnetic encoders are highly sturdy devices that can be used even in harsh environments. There are two types of magnetic encoders: incremental encoders, which count pulse trains; and sinusoidal encoders, which use analog signals. Incremental encoders consist of a magnetic drum and magnetic sensor, which convert changes in the magnetic flux generated by the rotation of the shaft into a pulse train in order to obtain information about the encoder's angle ${ }^{(4)}$. For this reason, it is not possible to calculate the absolute angle, which means that increasing the angular resolution requires a corresponding increase in the number of poles, and thus in size. Sinusoidal encoders, like sinusoidal resolvers, use a sinusoidally magnetized magnet to calculate the angle

\footnotetext{
* Chuo University

1-13-27, Kasuga, Bunkyo-ku, Tokyo 112-0003, Japan
}

from the orthogonal signals that correspond to the angle of rotation $^{(5)-(8)}$. While using a bipolar magnet makes it possible to obtain the absolute angle easily, the fact that the angular resolution depends on signal precision makes it difficult to achieve high angular resolution. Using signal processing to improve signal precision ${ }^{(9)(10)}$ and improving angular resolution with a multipolarized magnet ${ }^{(11)}$ have been found to improve the angular resolution. However, because multipolarization means that it is impossible to calculate the absolute angle, absolute encoders require additional mechanisms and would result in increased size.

In this paper, we propose an absolute magnetic encoder whose absolute angle can be calculated even when its magnet is multipolarized. The proposed magnetic encoder consists of only one magnet and four Hall elements-a structure whose simplicity makes it small and cost-efficient. The basic approach is to calculate the absolute angle using four signals that are distinguished through eccentric rotation. Furthermore, since these 4 signals can be used to obtain the 2 angles orthogonal to them (SIN and COS), this approach can be applied to existing methods of angle calculation that use converters. This makes it possible to calculate the absolute angle and attain the improved precision that increasing the number of magnetic poles would produce, which was difficult to do with existing methods.

\section{Conventional Magnetic Encoders}

Figure 1 shows a conventional magnetic encoder. Encoders with a bipolar magnet use Hall elements placed around the magnet $90^{\circ}$ apart to obtain the orthogonal signals.

$$
\begin{aligned}
& V_{\text {sin }}=\sin \left(\theta_{m}\right) \ldots \ldots \ldots \ldots \ldots \ldots \ldots \ldots \ldots \ldots \ldots \ldots \ldots \ldots \ldots \ldots \ldots \ldots \ldots \ldots \ldots \ldots \ldots \ldots \ldots
\end{aligned}
$$

There are various methods to calculate the angle from the orthogonal signals, though the most basic approach is to calculate it using the arctan of the two signals. 


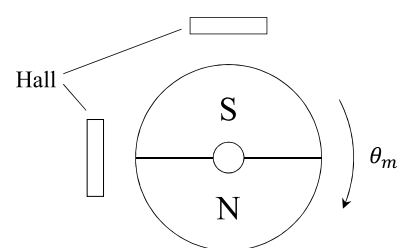

Fig. 1. Conventional magnetic encoder

$$
\theta_{m}=\arctan \left(\frac{\mathrm{V}_{\text {sin }}}{V_{c o s}}\right)
$$

For this reason, even though magnetic encoders that use a bipolar magnet, whose absolute angle can be calculated, it is difficult to attain precision above a certain level because the angular resolution depends on the precision of the orthogonal signals obtained by the Hall elements.

Improving the angular resolution requires increasing the number of poles in the magnet:

$$
\begin{aligned}
& V_{\text {sin }}=\sin \left(\theta_{e}\right) \\
& V_{c o s}=\cos \left(\theta_{e}\right) \\
& \theta_{e}=P \theta_{m} \ldots \ldots
\end{aligned}
$$

making the shaft of the angle, $\theta_{m}$,

$$
\begin{aligned}
& \theta_{e}=\arctan \left(\frac{V_{\text {sin }}}{V_{\text {cos }}}\right) \ldots \ldots \ldots \ldots \ldots \ldots \\
& \theta_{m}=\frac{\theta_{e}}{P}+\frac{2 n \pi}{P}(n=0,1, \ldots, P-1) .
\end{aligned}
$$

Where, $P$ is the number pole pairs in the magnet. Equation (8) shows that increasing the number of poles makes it impossible to calculate the absolute angle.

\section{The Proposed Magnetic Encoder}

3.1 Overview Figure 2 shows the proposed magnetic encoder. It consists of (a) a multipolar magnet that rotates eccentrically with the same period as the rotation of the shaft and (b) two pairs of Hall sensors around the magnet, with the sensors in each pair placed $45^{\circ}$ apart. The magnet used is a sinusoidally magnetized, 4-pole ring magnet. Using a multipolar magnet makes it possible to attain improved angular resolution. By using an eccentric structure, the proposed method makes it possible to produce a high-precision encoder with a simple composition, in comparison to existing research ${ }^{(5)-(8)(11)}$, and one that is easy to make small as well. While the eccentric rotation of the magnet generates oscillations through its unbalance force and may lead to degraded precision and a decreased lifespan, this issue can easily be solved by mounting a non-magnetic counterweight to keep the mass in equilibrium.

Figure 3 shows an overview of the angle calculation. $V_{s+}$, $V_{s-}, V_{c+}, V_{c-}$ are the changes in magnetic flux generated through the eccentric rotation of the magnet, as measured by each Hall element. Taking the sum of the signals that are in a $180^{\circ}$ relationship to each other yields the orthogonal signals. As in conventional systems, a converter is used to obtain the electrical degree $\theta_{e}$ from these orthogonal signals, and the offset $n$ is calculated by using the signals $V_{s+}, V_{s-}, V_{c+}, V_{c-}$ obtained by the Hall elements. This electrical degree $\theta_{e}$ and offset $n$ are combined to calculate the angle of the shaft $\theta_{m}$.

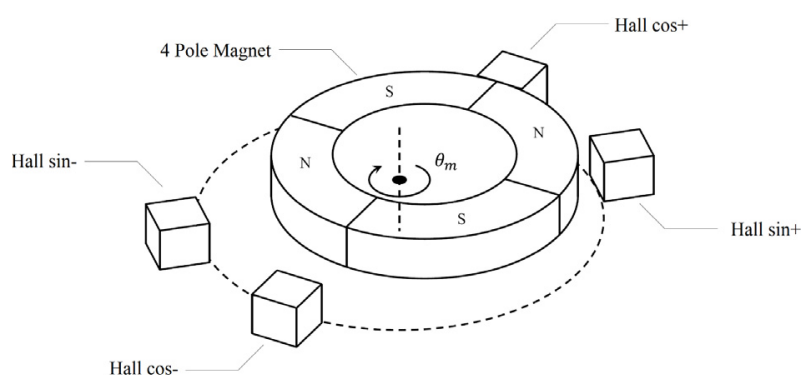

Fig. 2. Proposed magnetic encoder

Using four signals distinguished by the eccentric rotation of the magnet makes it possible to solve the issue of not being able to determine the offset $n$, which is a consequence of increasing the number of poles.

\subsection{Signals Distinguished through Eccentric Rotation}

Each Hall element outputs a signal that corresponds to the change in magnetic flux due to the eccentric rotation of the magnet. Figure 4 shows what happens when the angle of the shaft $\theta_{m}$ is rotated by $\theta$. Here, $r_{m}$ is the radius of the magnet, $a$ is the distance from the center of the magnet to the axis of rotation, $h_{i}=\left(r_{i}, \theta_{i}\right)(i=0,1,2,3)$ are the coordinates of each Hall element, and we let $r_{0}=r_{1}=r_{2}=r_{3}=r$, $\theta_{0}=0^{\circ}, \theta_{1}=180^{\circ}, \theta_{2}=-45^{\circ}, \theta_{3}=135^{\circ}$. If we let $d_{i}$ be shortest distance from the magnet to a given Hall element, then when the change in $d_{i}$ is small, the magnetic flux density $B_{i}$ generated by that Hall element can be approximated in inverse proportion to $d_{i}$, yielding

$$
B_{i}=B \frac{\sin (P \alpha)}{d_{i}+c}
$$

Where, $B$ is the amplitude of the magnetic flux density, $P$ is the number of pole pairs, and $c$ is a constant. $d_{i}$ and $\sin (P \alpha)$ change as the angle of the shaft $\theta_{m}$ changes. According to the law of cosines, $d_{i}$ is

$$
d_{i}=\sqrt{r_{i}^{2}+a^{2}-2 r_{i} a \cos \left(\theta_{m}-\theta_{i}\right)}-r_{m}
$$

and when the number of pole pairs $P=2, \sin (P \alpha)$ can be determined as follows:

$$
\begin{aligned}
& \sin (P \alpha)=\sin (2 \alpha)=2 \sin (\alpha) \cos (\alpha) \ldots \ldots \ldots \ldots \ldots \ldots \ldots \ldots \ldots \ldots \ldots \\
& \sin (\alpha)=\frac{r_{i}}{d_{i}+r_{m}} \sin \left(\theta_{m}-\theta_{i}\right) \ldots \ldots \ldots \ldots \ldots \\
& \cos (\alpha)=\frac{1}{2 a\left(d_{i}+r_{m}\right)}\left\{a^{2}+\left(d_{i}+r_{m}\right)^{2}-r_{i}^{2}\right\} \cdots \cdots
\end{aligned}
$$

Figure 5 shows the signals distinguished through eccentric rotation when we let $r=8.3[\mathrm{~mm}], r_{m}=7.5[\mathrm{~mm}]$, $a=0.5[\mathrm{~mm}]$. However,

$$
\begin{aligned}
& V_{s+}=V_{0}=B_{0} \\
& V_{s-}=V_{1}=B_{1} \\
& V_{c+}=V_{2}=B_{2} \\
& V_{c-}=V_{3}=B_{3}
\end{aligned}
$$

and adding the pairs of signals together yields

$$
\begin{aligned}
& V_{s}=V_{s+}+V_{s-} \\
& V_{c}=V_{c+}+V_{c-}
\end{aligned}
$$




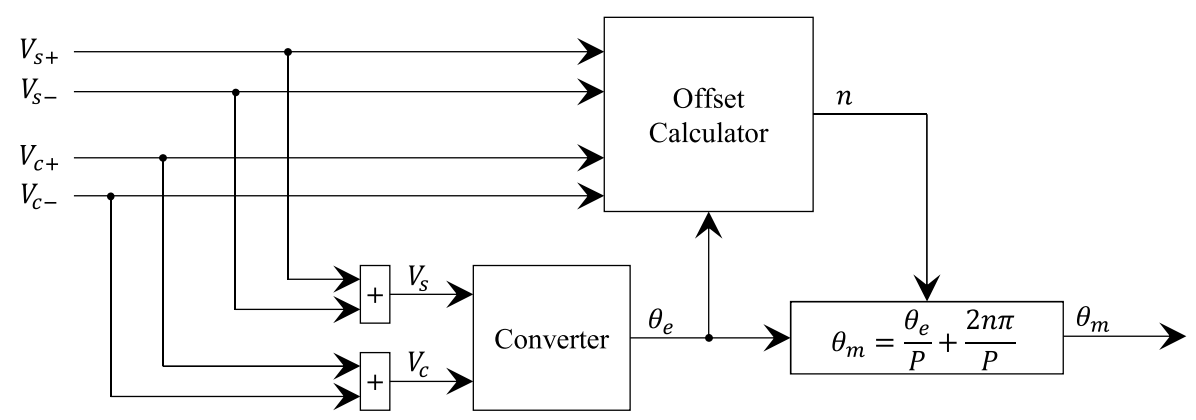

Fig. 3. Diagram of signal processing

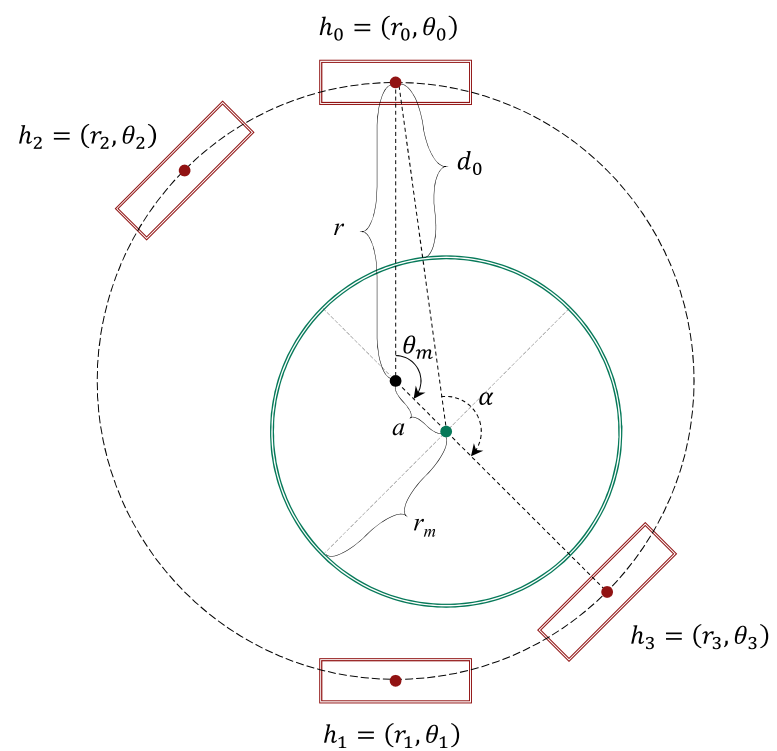

Fig. 4. Analytical model

which is shown in Fig. 6. In this way, it is possible to generate orthogonal signals from the signals distinguished through eccentric rotation and thus we can use existing methods to calculate the angle of the shaft.

3.3 Converter As mentioned in Section 3.2, it is possible to generate orthogonal signals from the signals distinguished through eccentric rotation. This is essentially why existing methods of calculating the angle can be used to configure a converter. Many methods have been proposed to calculate the angle from orthogonal signals ${ }^{(12)-(15)}$, and in this paper, we will do so using linear sectors of the signals. As Fig. 7 shows, $V_{o}$ is made up of whichever of the orthogonal signals $V_{s}, V_{c}$ is closer to 0 at any point, and is thus the result of extracting linear sectors of the orthogonal signals $V_{s}, V_{c}$. After dividing the signals into four sectors, as Fig. 7 shows, the relationship between $V_{o}$ and the electrical degree $\theta_{e}$ in each sector is measured offline ahead of time and saved in a lookup table for that sector. There will be phase offset and waveform distortion in the signals obtained by the Hall elements depending on how precisely they are installed and how precisely the axis of rotation is mounted. However, it is possible to counteract this by using including phase offset and waveform distortion in the lookup table. Though this will increase required memory capacity, it will make the completed sectors shorter, which reduces calculation errors and increases precision. Using such a lookup table makes it possible to calculate

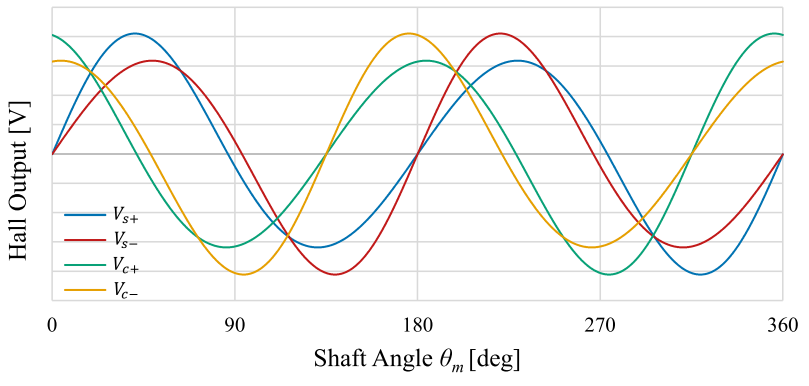

Fig. 5. Simulation of output signals

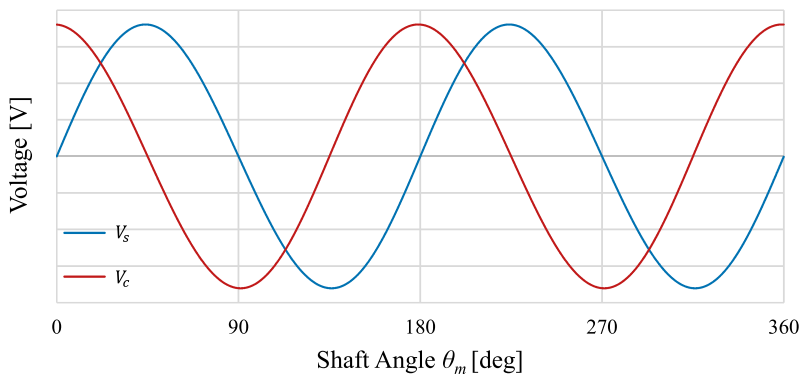

Fig. 6. Simulation of quadrature signals

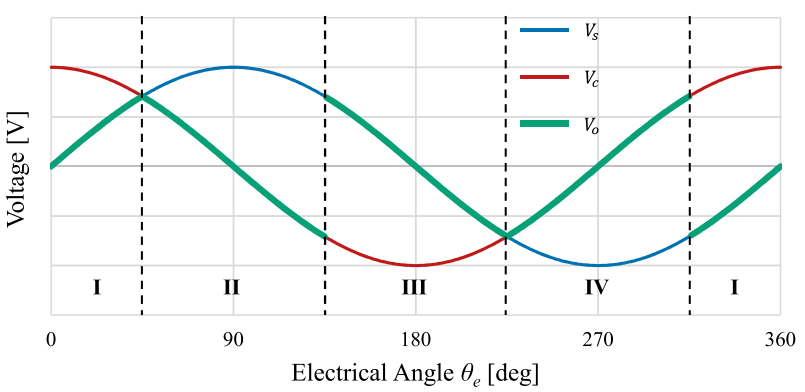

Fig. 7. Divided signals into four sectors

the electrical degree $\theta_{e}$ directly from $V_{s}, V_{c}$.

Figure 8 shows an overview of the converter. The input orthogonal signals $V_{s}, V_{c}$ are identified using the sectors in the lookup tables. To do so, we use Table 1 as the basis for identification. After that, the chosen lookup table is used to calculate the electrical degree $\theta_{e}$.

3.4 Calculating Offset The relationship between the electrical degree $\theta_{e}$ obtained by the converter and the angle of the shaft $\theta_{m}$ is expressed in equation (8). This equation means that when the angle of the shaft $\theta_{m}$ is calculated from $\theta_{e}$, the electrical degree obtained by the converter, it is not possible determine $n$, for which there are $P-1$ possibilities. However, $n$ can be determined using the four signals 


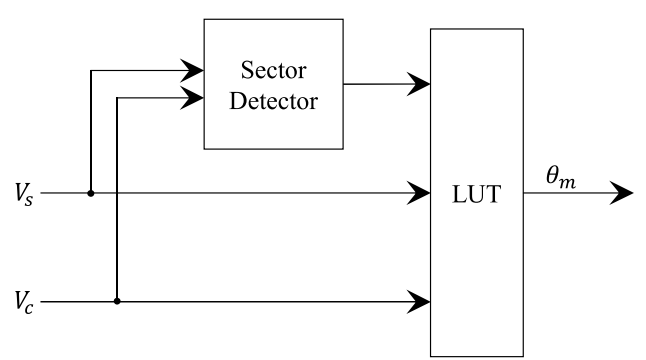

Fig. 8. Diagram of converter

Table 1. Sammary of sector detector

\begin{tabular}{ccc}
\hline Condition1 & Condition2 & Sector \\
\hline$\left|V_{s}\right|<\left|V_{c}\right|$ & $V_{c}>0$ & I \\
$\left|V_{s}\right|>\left|V_{c}\right|$ & $V_{s}>0$ & II \\
$\left|V_{s}\right|<\left|V_{c}\right|$ & $V_{c}<0$ & III \\
$\left|V_{s}\right|>\left|V_{c}\right|$ & $V_{s}<0$ & IV \\
\hline
\end{tabular}

distinguished through eccentric rotation. As Fig. 6 shows, in $0^{\circ} \leq \theta<180^{\circ}$, at least one of the signals $V_{s+}, V_{s-}, V_{c+}, V_{c-}$ satisfies $V(\theta) \neq V\left(\theta+180^{\circ}\right)$. We can take advantage of this fact and use the method of least squares to determine $n$.

We will save the relationships between $V_{s+}, V_{s-}, V_{c+}, V_{c-}$ and the angle of the shaft $\theta_{m}$ in the lookup tables ahead of time offline. We will use these lookup tables to calculate the sum of squared errors with respect to all $n$ as follows:

$$
J_{n}=\sum_{i=0}^{3}\left(V_{i}-f_{i}\left(\frac{\theta_{e}}{P}+\frac{2 n \pi}{P}\right)\right)^{2} \ldots \ldots \ldots \ldots \ldots \ldots
$$

Where, $V_{i}$ are the signals distinguished through eccentric rotation and $f_{i}$ are the lookup tables. By choosing the $n$ that minimizes $J_{n}$, we can calculate the absolute angle.

To calculate the offset, there must be a characteristic amount of eccentricity generated in $V_{s+}, V_{s-}, V_{c+}, V_{c-}$. This characteristic amount is determined by the ratio of the amount of eccentricity $a$ to the radius of the magnet $r_{m}, \frac{a}{r_{m}}$. On the other hand, an increase in the amount of eccentricity $a$ leads directly to signal distortion. For this reason, it is not enough to simply choose a large value; we must consider the precision of the signal in order to find an amount of eccentricity $a$ that will allow the offset to be calculated correctly.

3.5 Precision If we suppose that when the orthogonal signal changes from minimum to maximum, the A/D value changes from $-2^{N-1}$ to $2^{N-1}$, then $-v<V<v$, the range of the orthogonal signals that is used in angle calculation, are the values of each sector up to the point where it switches over. This means that

$$
v=2^{N-1} \times \sin \left(45^{\circ}\right)=2^{N-\frac{3}{2}} .
$$

Where, $N$ is the number of bits of the $\mathrm{A} / \mathrm{D}$ angular resolution. There are $4 \times P$ of these sectors, which means that the angular resolution $R$ of angle calculation is

$$
\begin{aligned}
R & =2 \times 2^{N-\frac{3}{2}} \times 4 \times P \\
& =P \times 2^{N+\frac{3}{2}} \ldots \ldots \ldots
\end{aligned}
$$

If we also define the change in the angle when $\mathrm{A} / \mathrm{D}$ changes by 1 [LSB] as the precision $A$, then the precision $A$ reaches its maximum where the slope of the linear sector is at its minimum, and the precision $A$ at that point will be the reciprocal of the slope of the linear sector. Because the slope of each linear sector is at its minimum at its switch-over point,

$$
\begin{aligned}
\frac{1}{A} & =2^{N-1} \times \sin ^{\prime}\left(P \theta_{m}\right) \\
& =2^{N-1} \times P \cos \left(45^{\circ}\right) \\
\therefore A & =\frac{1}{2^{N-\frac{3}{2}} P}[\mathrm{rad}] \cdots \cdots
\end{aligned}
$$

This shows that the improvement in angular resolution and precision is related to the number of pole pairs $P$.

\section{Experiment}

To evaluate the proposed magnetic encoder, we made one configured as described above. Hall ICs were used to measure the change in magnetic flux caused by the eccentric rotation of the magnet, and their output voltage was converted to digital signals using AD converters with 10 [bit] angular resolution. For the Hall ICs, we used Allegro MicroSystems A1324 and for the AD converter, we used Analog Devices AD7904. With the A/D precision as a reference, we used a simulation to determine an appropriate amount of eccentricity $a$ that would enable us to calculate the offset. The specifications of our magnetic encoder are shown in Table 2. To evaluate it, we connected an incremental optical encoder with an angular resolution of 14400 to it. Figure 9 shows the setup used for evaluation.

Figure 10 shows the relationship between the signals distinguished through eccentric rotation, as measured by the Hall ICs, and the angle of the shaft $\theta_{m}$. We obtained signals that were similar to Fig. 5, which were derived from our theoretical formula. Figure 11 shows the orthogonal signals we obtained by adding each signal pair together, which are likewise similar to the theoretically-derived ones in Fig. 6. This indicates that the approximation in equation (9) holds true when an appropriate amount of eccentricity is designated.

Figure 12 shows (a) the relationship between the calculated angle of the shaft $\theta_{\text {calc }}$ and the angle of the shaft as measured

Table 2. Parameter of magnetic encoder

\begin{tabular}{ccc}
\hline Parameter & Unit & Value \\
\hline$r_{0}$ & $\mathrm{~mm}$ & 8.3 \\
$r_{1}$ & $\mathrm{~mm}$ & 8.3 \\
$r_{2}$ & $\mathrm{~mm}$ & 8.3 \\
$r_{3}$ & $\mathrm{~mm}$ & 8.3 \\
$\theta_{0}$ & degree & 0 \\
$\theta_{1}$ & degree & 180 \\
$\theta_{2}$ & degree & -45 \\
$\theta_{3}$ & degree & 135 \\
$r_{m}$ & mm & 7.5 \\
$a$ & $\mathrm{~mm}$ & 0.5 \\
\hline
\end{tabular}

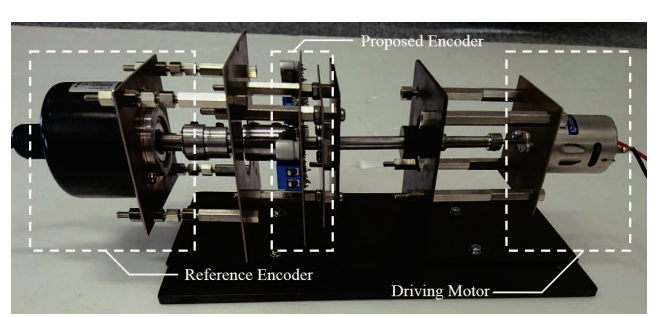

Fig. 9. Experiment setup 


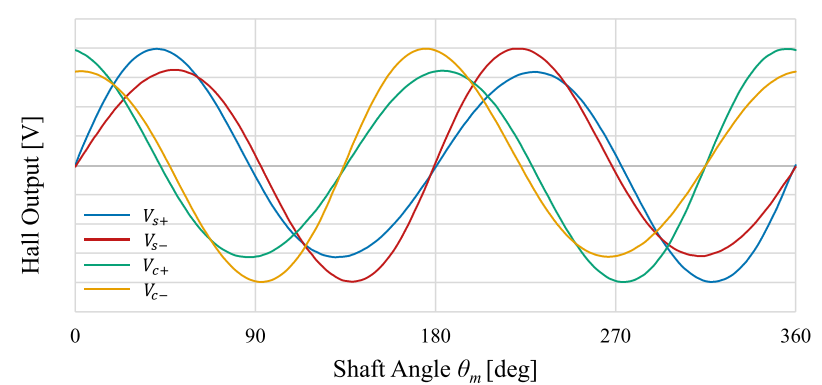

Fig. 10. Output signals from hall elements

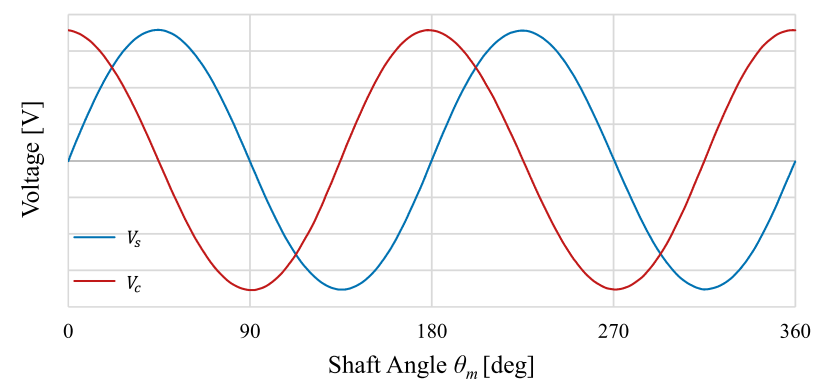

Fig. 11. Quadrature signals

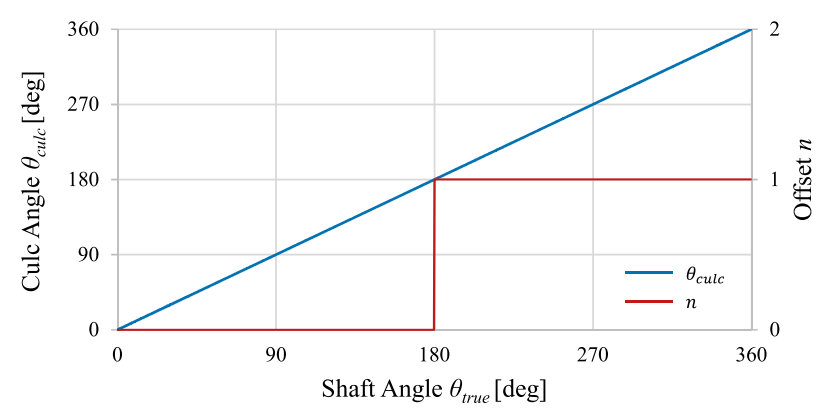

Fig. 12. Result of calculation angle

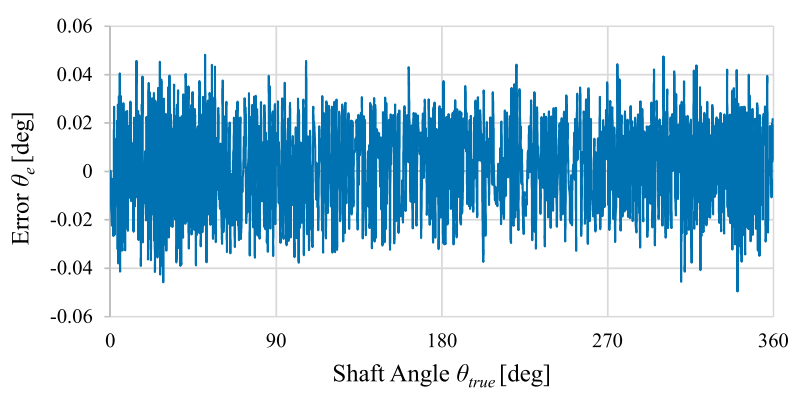

Fig. 13. Error of magnetic encoder

by the optical encoder $\theta_{\text {true }}$ and (b) the relationship between the offset $n$ and the angle of the shaft as measured by the optical encoder $\theta_{\text {true }}$. This shows that the offset $n$ can be accurately determined from the signals distinguished through eccentric rotation, which is then used to calculate the absolute angle. In addition, Fig. 13 shows the angle calculation error $\theta_{e}=\theta_{\text {calc }}-\theta_{\text {true }}$. Equation (24) suggests that we achieved a precision $A$ of approximately $0.08[\mathrm{deg}]$ with the parameters used in this experiment. Thus, we can say that we attained a precision that is almost equal to the precision stated in Section 3.5. This result also shows that the proposed encoder attains a precision of over 12 bits.

\section{Conclusion}

In this paper, we have proposed a new magnetic encoder that uses an eccentric structure to enable calculation of the absolute angle even with a multipolarized magnet, in order to attain improved angular resolution. The proposed magnetic encoder consists of only one magnet and four Hall elements-a structure whose simplicity makes it small and cost-efficient. Its absolute angle can be calculated using four signals distinguished through the eccentric rotation of the magnet. Experimental evaluation of the performance of the proposed method showed that it is possible to calculate the absolute angle and that is possible to produce a high-precision magnetic encoder by using an eccentric structure.

\section{Acknowledgment}

This research was supported by a Chuo University Collaborative Research Grant.

\section{References}

( 1 ) T. Kojima, Y. Kikuchi, S. Seki, and H. Wakiwaka: "Study on High Accuracy Optical Encoder with 30 bits", in Proc. The 8th Workshop IEEE Advanced Motion Control, pp.493-498 (2004)

( 2 ) R. Sawada, H. Tanaka, O. Ohguchi, J, Shimada, and S. Hara: "Fabrication of Active Integrated Optical Micro-Encoder", in Proc. IEEE Micro Electro Mechanical Systems, pp.233-238 (1991)

( 3 ) A. Waheed and L. Cai: "Alternative Design for Optical Incremental Encoder Mesurement Systems", in Proc. IEEE International Conference on Industrial Technology, pp.634-639 (2016)

( 4 ) Y. Kikuchi, F. Nakamura, H. Wakiwaka, and H. Yamada: "Consideration for a High Resolution of Magnetic Rotary Encoder", IEEE Transactions on Magnetics, Vol.32, No.5, pp.4959-4961 (1996)

( 5 ) S.-H. Jeong, S.-H. Rhyu, B.I. Kwon, and B.-T. Kim: "Design of the Rotary Magnetic Position Sensor With the Sinusoidally Magnetized Permanent Magnet", IEEE Transactions on Magnetics, Vol.43, No.4 (2007)

( 6 ) T. Kabashima, Y. Arinaga, K. Uemura, I. Murokita, and M. Ohto: "A Novel Magnetic Rotary Encoder for Servo Motors", IEEJ Transactions on Industry Applications, Vol.126, No.9, pp.1202-1207 (2006)

( 7 ) S. Hao, Y. Liu, and M. Hao: "Study on a Novel Absolute Magnetic Encoder", in Proc. IEEE International Conference on Robotics and Biomimetics, pp.1773-1776 (2009)

( 8 ) S.-T. Wu, J.-Y. Chen, and S.-H. Wu: "A Rotary Encoder With an Eccentrically Mounted Ring Magnet", IEEE Transactions on Instrumentation and Measurement, Vol.63, No.8, pp.1907-1915 (2014)

( 9 ) H.V. Hoang and J.W. Jeon: "Signal Compensation and Extraction of High Resolution Position for Sinusoidal Magnetic Encoders", in Proc. IEEE International Conference on Control, Automation and Systems, pp.1368-1373 (2007)

(10) S.H. Hwang, J.H. Lee, and J.M. Kim: "Compensation of Analog Rotor Position Error due to Nonideal Sinusoidal Encoder Output Signals", in Proc. IEEE Energy Conversion Congress and Exposition, pp.4469-4473 (2010)

(11) Y. Liu, S. Hao, and M. Hao: "A Novel Absolute Magnetic Encoder based on Pseudorandom Code", in Proc. IEEE International Conference on Information and Automation, pp.385-390 (2009)

(12) C.-H. Yim, I.-J. Ha, and M.-S. Ko: "A Resolver-to-Digital Conversion Method for Fast Tracking", IEEE Transactions Industiral Electronics, Vol.39, No.5 (1992)

(13) L. Ben-Brahim, M. Benammar, M.A. Alhamadi, N.A. Al-Emadi, and M.A. Al-Hitmi: “A New Low Cost Linear Resolver Converter", IEEE Sensors Journal, Vol.8, No.10 (2008)

(14) H.T. Le, H.V. Hoang, and J.W. Jeon: "Efficient Method for Correction and Interpolation Signal of Magnetic Encoders", in Proc. IEEE International Conference on Industrial Informatics (2008)

(15) M. Benammar, L. Ben-Brahim, and M.A. Alhamadi: "A Novel Resolver-to$360^{\circ}$ Linearized Converter”, IEEE Sensors Journal, Vol.4, No.1 (2004) 
Kodai Yamamoto (Non-member) received the B.Sc.Eng. degrees in

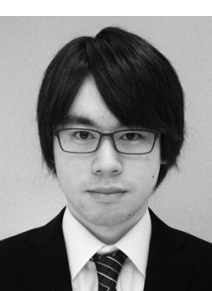
electrical, electronic, and communication engineering from the Chuo University in 2016. He is studying electrical, electronic, and communication engineering at the postgraduate program of the Chuo University. His interests are in Control, Robotics, Intelligent Spaces.

\section{Kazuki Otomo}

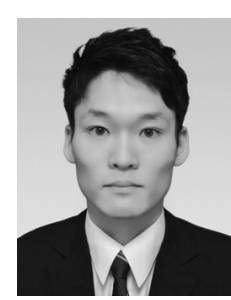

(Non-member) received the B.Sc.Eng. degrees in electrical, electronic, and communication engineering from the Chuo University in 2017. He is studying electrical, electronic, and communication engineering at the postgraduate program of the Chuo University. His interests are in Control, Robotics, Intelligent Spaces.
Hideki Hashimoto (Senior Member) received the B.E., M.E., and

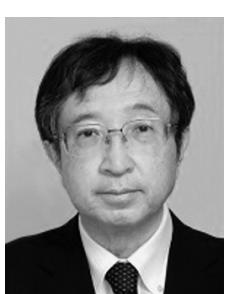
Dr.Eng. degrees in electrical engineering from the University of Tokyo in 1981, 1984, and 1987, respectively. He joined the Institute of Industrial Science, University of Tokyo, as a Lecturer in 1987, and was an Associate Professor from 1990 to 2011. He has been a Professor at Chuo University since 2011. His interests are in Control, Robotics, Intelligent Spaces and AI. He is a fellow of IEEE, SICE and RSJ. 